11,05

\title{
Фазовые переходы в двумерной слабо разбавленной пятивершинной модели Поттса
}

\author{
() А.К. Муртазаев ${ }^{1,2}$, А.Б. Бабаев ${ }^{1,3}$, Г.Я. Атаева ${ }^{1,9}$ \\ ${ }^{1}$ Институт фризики им. Х.И. Амирханова ДФИЦ РАН, \\ Махачкала, Россия \\ 2 Дагестанский государственный университет, \\ Махачкала, Россия \\ ${ }^{3}$ Дагестанский государственный педагогический университет, \\ Махачкала, Россия \\ ฯ E-mail: ataeva20102014@mail.ru
}

Поступила в Редакцию 26 февраля 2020 г.

В окончательной редакции 26 февраля 2020 г.

Принята к публикации 12 марта 2020 г.

\begin{abstract}
Методом компьютерного моделирования проведено исследование фазовых переходов в двумерной слабо разбавленной модели Поттса на квадратной решетке при $q=5$. Рассмотрены системы с линейными размерами $L \times L=N, L=10-120$. На основе кумулянтов Биндера четвертого порядка и методом гистограммного анализа данных, показано, что введение немагнитных примесей в спиновую систему, описываемой двумерной моделью Поттса с $q=5$, приводит к смене фазового перехода первого рода на фазовый переход второго рода
\end{abstract}

Ключевые слова:примесь, метод Монте-Карло, магнитная система, модель Поттса.

DOI: $10.21883 /$ FTT.2020.07.49478.051

\section{1. Введение}

В настоящее время хорошо известно, что присутствие немагнитных примесей и дефектов структуры оказывает влияние на тепловые и магнитные характеристики систем, и в частности оказывает существенное влияние на поведение систем при фазовых переходах (ФП), если критический индекс теплоемкости соответствующий чистой системе положителен, т.е. $\alpha>0$. В противоположном случае, когда $\alpha<0$, слабый беспорядок не влияет на критическое поведение (критерий Харриса [1]). В то же время имеются основания предполагать, что примеси оказывают совершенно другое влияние вплоть до изменения рода ФП в случае спиновых систем, испытывающих в однородном состоянии ФП первого рода [2].

В настоящей работе исследованы ФП в двумерной слабо разбавленной модели Поттса с числом состояний спина $q=5$ на квадратной решетке при концентрации спинов $p=1.00,0.90,0.80$. Исследования проведены на основе кластерного алгоритма Вольфа метода МонтеКарло (МК). Касательно двумерной разбавленной модели Поттса с $q=5$, до сих пор нет достоверных данных о влиянии немагнитных примесей на тепловые и магнитные свойства, не исследовано их влияние на фазовые переходы, нет сведений о зависимости критических индексов от концентрации немагнитных примесей, особенно когда беспорядок реализован в виде вмороженных немагнитных примесей [3]. Единственным надежно установленным фактом является, то, что в чистой модели происходит ФП первого рода согласно аналитическим методам [4].
Гамильтониан двумерной слабо разбавленной модели Поттса может быть, представлен в следующем виде [4]:

$$
H=-\frac{J}{2} \sum_{i, j} \rho_{i} \rho_{j} \cos \Theta_{i, j},
$$

$\theta_{i, j}-$ угол между взаимодействующими спинами $S_{i}-S_{j}$, где $J-$ параметр обменного ферромагнитного взаимодействия ближайших соседей, $\rho_{i}=1$, если узел $i$ занят магнитным атомом, и $\rho_{i}=0$, если в $i$-узле немагнитная примесь. Исследовались системы с линейными размерами $L \times L=N, L=10 \div 120$.

\section{2. Результаты численного эксперимента}

Для анализа характера фазового перехода был использован метод кумулянтов Биндера четвертого порядка [5]:

$$
\begin{gathered}
V_{L}(T, p)=1-\frac{\left\langle E^{4}(T, p ; L)\right\rangle_{L}}{3\left\langle E^{2}(T, p ; L)\right\rangle_{L}^{2}}, \\
U_{L}(T, p)=1-\frac{\left\langle m^{4}(T, p ; L)\right\rangle_{L}}{3\left\langle m^{2}(T, p ; L)\right\rangle_{L}^{2}},
\end{gathered}
$$

где $E-$ энергия и $m-$ намагниченность системы с линейным размером $L$. Выражения (2) и (3) позволяют определить $T_{c}(p)$ с большой точностью в фазовых переходах первого и второго рода соответственно. Данный метод, хорошо зарекомендовал себя и при определении рода ФП [6-10]. Характерные зависимости кумулянтов 


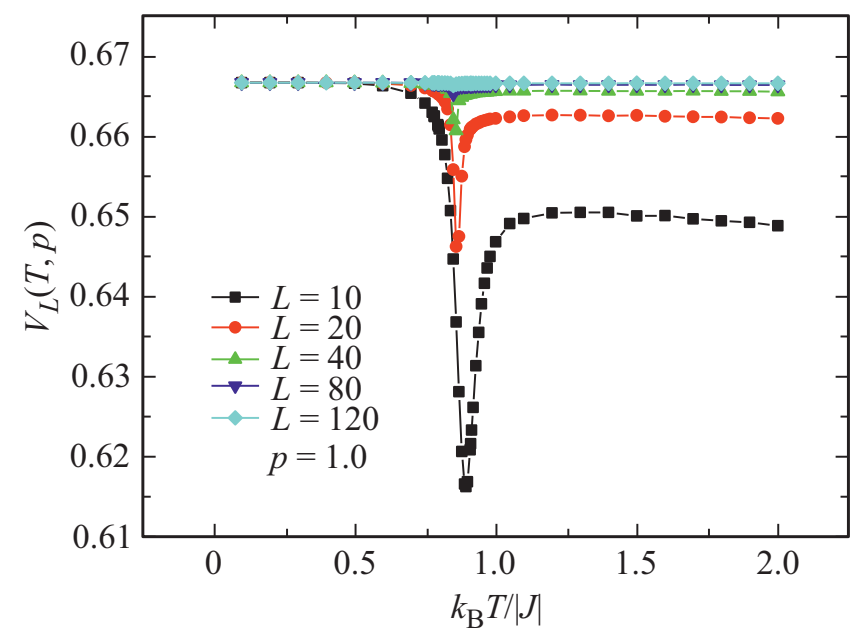

Рис. 1. Температурная зависимость кумулянтов Биндера $V_{L}(T, p)$ для модели Поттса при $p=1.00$.

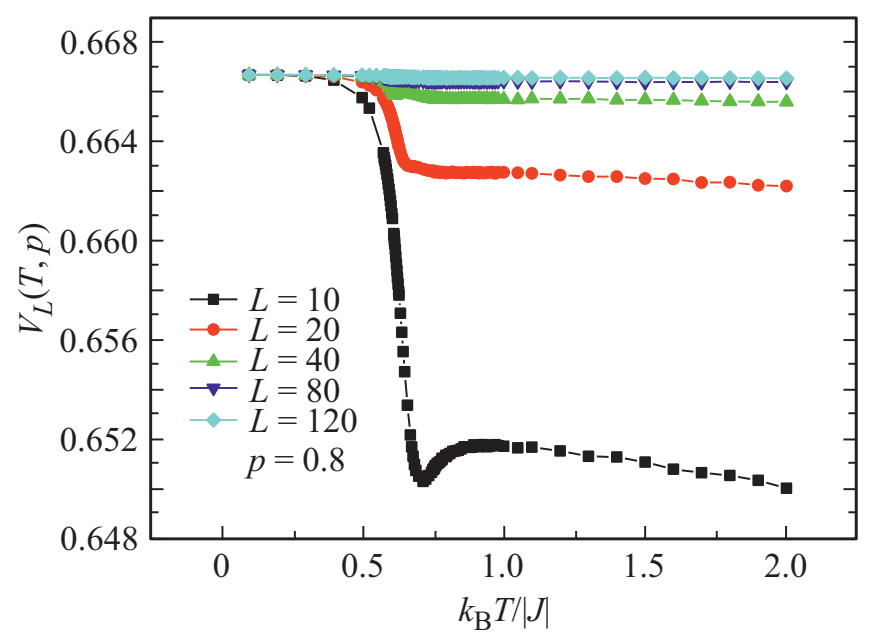

Рис. 2. Температурная зависимость кумулянтов Биндера $V_{L}(T, p)$ слабо разбавленной модели Поттса при $p=0.80$.

Биндера $V_{L}(T, p)$ от температуры для систем с разными линейными размерами при $p=1.00$ и $p=0.80$ приведены на рис. 1 и 2 соответственно. На рис. 2 наглядно видно, что нетривиальная величина $V^{*} \rightarrow 2 / 3$ в соответствии с выражением $V(T, p)=V^{*}+b L^{-d}$ при $L \rightarrow \infty$. Такое поведение как отмечалось выше характерно для ФП второго рода. На рис. 3 и 4 приведены зависимости кумулянтов Биндера $U_{L}(T, p)$ от температуры при $p=1.00$ и $p=0.80$. На рис. 4 видно, что в критической области для $U_{L}(T, p)$ наблюдается четко выраженная точка пересечения и $U_{L}(T, p)$ не проявляет тенденцию стремления к $-\infty$ при $L \rightarrow \infty$, что также свидетельствует о ФП второго рода. Полученные таким способом температуры фазовых переходов $T_{l}(p)$ в единицах $|J| / k_{B}$ равны: $T_{l}(1.0)=0.8515(1)$, $T_{l}(0.90)=0.731(2), T_{l}(0.80)=0.57(2)$. Как видно, температура ФП полученная для чистой спиновой системы при $p=1.0$ достаточно хорошо согласуется с аналити- ческим значением, полученным Бакстером [4]по формуле $\frac{k_{B} T_{L}}{|J|}=\frac{1}{\ln (1+\sqrt{5})}=0.8515$.

Кроме кумулянтов Биндера для анализа рода ФП нами использовался и гистограммный анализ данных метода МК [11-13]. Гистограммный анализ данных проведенный нами для двумерной чистой ферромагнитной модели Поттса с числом состояний спина $q=5$ на квадратной решетке также свидетельствует о наличии ФП первого рода. Это продемонстрировано на рис. 5. На этом рисунке представлена гистограмма распределения энергии вблизи точки фазового перехода $T_{l}$ для систем с линейным размером $L=60$. Как видно из рисунка, на зависимости вероятности $P$ от энергии системы $U$ для системы $L=60$ наблюдается два хорошо выраженных максимума. Наличие бимодальности в распределении энергии является важным признаком ФП первого рода. Соответствующий гистограммный анализ данных был проведен и для двумерной слабо разбавленной ферро-

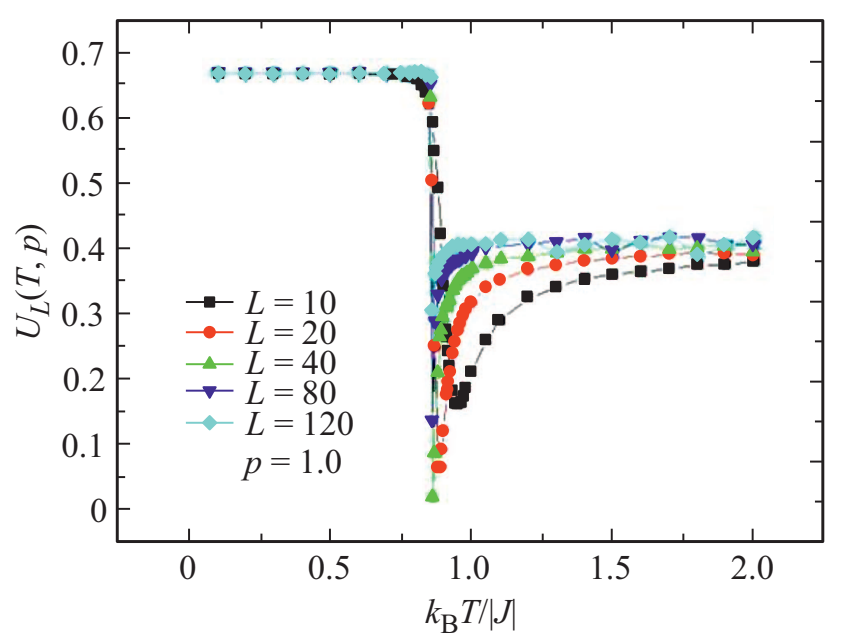

Рис. 3. Температурная зависимость кумулянтов Биндера $U_{L}(T, p)$ для модели Поттса при $p=1.00$.

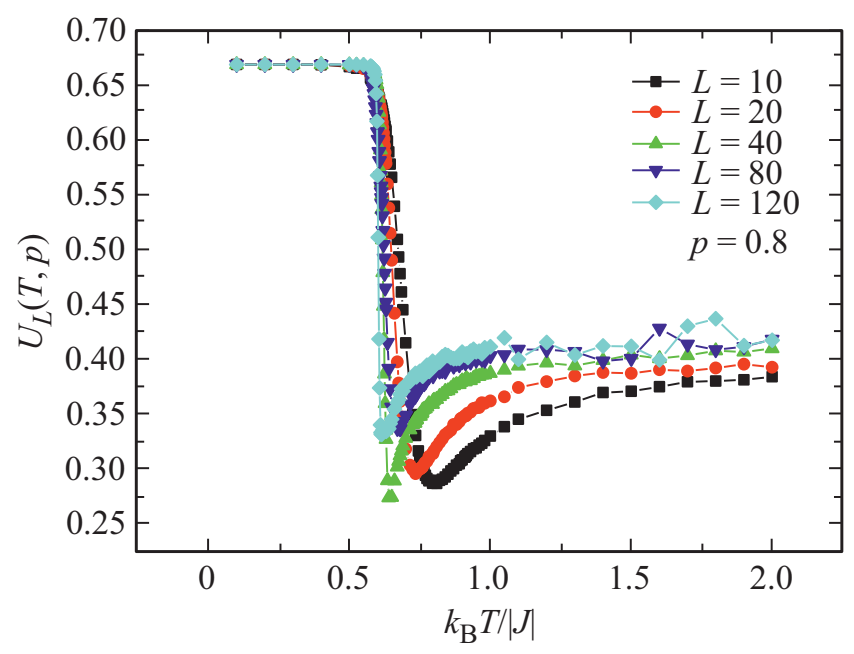

Рис. 4. Температурная зависимость кумулянтов Биндера $U_{L}(T, p)$ слабо разбавленной модели Поттса при $p=0.80$. 


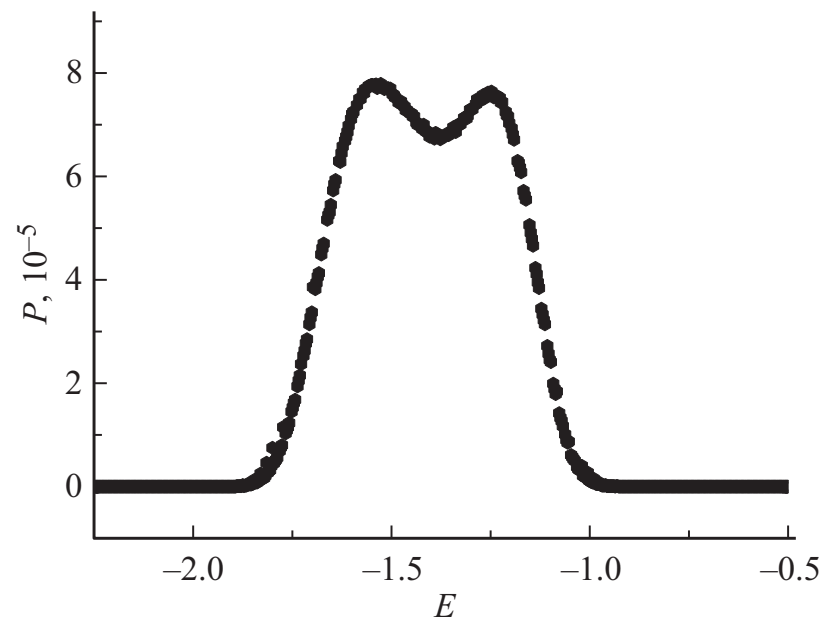

Рис. 5. Гистограмма распределения энергии для двумерной модели Поттса с $q=5$ при $p=1.0$.

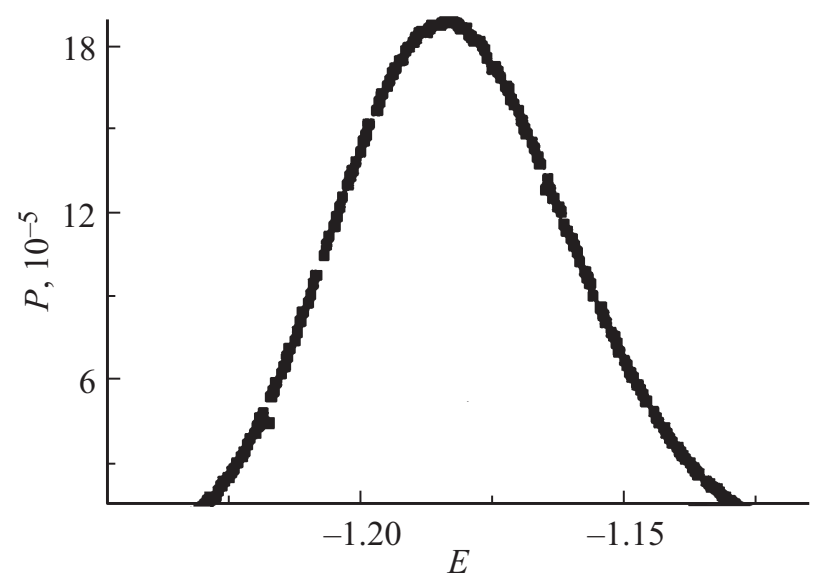

Pис. 6. Гистограмма распределения энергии для двумерной слабо разбавленной модели Поттса с $q=5$ при концентрации спинов $p=0.80$.

магнитной модели Поттса на квадратной решетке, но бимодальность в гистограмме распределения энергии для этой модели обнаружить не удалось. В этом случае в зависимости вероятности Р от энергии системы U для системы с $L=120$ наблюдается один хорошо выраженный максимум (см. рис. 6), что является характерным признаком для ФП второго рода.

\section{3. Заключение}

Таким образом, полученные данные свидетельствуют о том, что в двумерной ферромагнитной модели Поттса с $q=5$ в отсутствии структурного беспорядка происходит ФП первого рода в соответствии с предсказаниями теоретических методов [4]. Внесение вмороженного беспорядка $(c=1-p)$ в виде немагнитных примесей, каноническим способом, в рассматриваемую модель, приводит к смене ФП первого рода на ФП второго рода.

\section{Фининсирование}

Работа выполнена при финансовой поддержке РФФИ и рамках научного проекта № 18-32-20098 мол_а_вед.

\section{Список литературы}

[1] A.B. Harris. J. Phys. C 7, 1671 (1974).

[2] M. Aizenman. J. Wehr. Phys. Rev. Lett. 62, 2503 (1989).

[3] X. Qian, Y. Deng, W.J. Blöte. Phys. Rev E 72, 056132-1 (2005).

[4] F.Y. Wu. Exactly Solved Models: A Journey in Statistical Mechanics World Scientific, London (2009).

[5] K. Eichhorn, K. Binder. J. Phys.: Condens. Matter 8, 5209 (1996).

[6] A.K. Murtazaev, A.B. Babaev, M.A. Magomedov, F.A. Kassan-Ogly, A.I. Proshkin. JETP Lett. 100, 242 (2014).

[7] А.Б. Бабаев, А.К. Муртазаев. Физика низких температур 41, 8, 784 (2015).

[8] А.Б. Бабаев, А.К. Муртазаев. ФТТ 61, 7, 1342 (2019).

[9] A.K. Murtazaev, A.B. Babaev, G.Ya. Ataeva. Phys. Solid State 59, 1, 141 (2017).

[10] А.К. Муртазаев, А.Б. Бабаев, М.А. Магомедов, Ф.А. Кассан-Оглы, А.И. Прошкин. Письма в ЖЭТФ 100, 3, 267 (2014).

[11] А.Б. Бабаев, М.А. Магомедов, А.К. Муртазаев, Ф.А. Кассан-Оглы, А.И. Прошкин. ЖЭТФ 149, 2, 357 (2014).

[12] А.К. Муртазаев, А.Б. Бабаев. Письма в ЖЭТФ 99, 618 (2014).

[13] N.A. Alves, B.A. Berg, R. Villanova. Phys. Rev. B 41, 383 (1990).

Редактор Т.Н. Василевская 